\title{
Approximate analytic solution of fractional heat-like and wave-like equations with variable coefficients using the differential transforms method
}

\section{Aydin Secer ${ }^{*}$}

"Correspondence:

asecer@yildiz.edu.tr Department of Mathematical Engineering, Faculty of Chemical and Metallurgical Engineering, Yildiz Technical University, Davutpasa, istanbul, 34210, Turkey

\section{Springer}

\begin{abstract}
This paper uses the differential transform method (DTM) to obtain analytical solutions of fractional heat- and wave-like equations with variable coefficients. The time fractional heat-like and wave-like equations with variable coefficients were obtained by replacing a first-order and a second-order time derivative by a fractional derivative of order $0<\alpha<2$. The approach mainly rests on the DTM which is one of the approximate methods. The method can easily be applied to many problems and is capable of reducing the size of computational work. Some examples are presented to show the efficiency and simplicity of the method.
\end{abstract}

Keywords: wave-like equations; heat-like equations; differential transform method; fractional calculus

\section{Introduction}

Fractional order partial differential equations, as generalizations of classical integer order partial differential equations, have been used to model problems in fluid flow and other areas of application. Many phenomena in engineering physics, chemistry, and other sciences can be described very successfully by models using mathematical tools. Fractional derivatives provide an excellent instrument for the descriptive and hereditary properties of various materials and processes. In order to formulate certain electrochemical problems, half-order derivatives and integrals are more useful than the classical models [1]. Fractional differentiation and integration operators were also used for extensions of diffusion and wave equations [2].

Wazwaz and Gorguis [3] used the Adomian decomposition method for solving heatlike and wave-like models with variable coefficients. Momani [4] applied the method to the time fractional heat-like and wave-like equations with variable coefficients. The main disadvantage of the Adomian method is that the solution procedure for calculation of Adomian polynomials is complex and difficult as pointed out by many researchers [5-9]. $\mathrm{Xu}$ and Cang [10] solved the fractional heat-like and wave-like equations with variable coefficients using the homotopy analysis method (HAM). In 1998, the variational iteration method (VIM) was first proposed to solve fractional differential equations with great success [11]. Shou and He [12] used the VIM to solve various kinds of heat-like and wave-

(c) 2012 Secer; licensee Springer. This is an Open Access article distributed under the terms of the Creative Commons Attribution License (http://creativecommons.org/licenses/by/2.0), which permits unrestricted use, distribution, and reproduction in any medium, provided the original work is properly cited. 
like equations, and it was claimed that by using the variational iteration method, demerits of complex calculation of Adomian polynomials were omitted [12]. Recently, Molliq, Noorani, and Hashim [13] applied the VIM to solve various kinds of fractional heat-like and wave-like equations. The differential transform method (DTM) was first applied to engineering problems [14].

The DTM is a numerical method based on the Taylor series expansion which constructs an analytical solution in the form of a polynomial. The traditional high-order Taylor series method requires symbolic computation. However, the DTM obtains a polynomial series solution by means of an iterative procedure. Recently, the application of DTM is successfully extended to obtain analytical approximate solutions to ordinary differential equations of fractional order [15]. Application of fractional calculus in physics was presented by Hilfer [16]. A comparison between the VIM and the Adomian decomposition method for solving fractional differential equations is given by Odibat [17]. Recently, Kurulay [18] demonstrated the application of DTM for solving fmKdV. In this letter, we will apply the DTM [19] to fractional heat-like and wave-like equations to show the simplicity and straightforwardness of the method [20,21].

In this paper, we will consider the fractional heat-like and wave-like equations of the form [4]

$$
\begin{aligned}
\frac{\partial^{\alpha} u}{\partial t^{\alpha}} & =f(x, y, z) u_{x x}+g(x, y, z) u_{y y}+h(x, y, z) u_{z z}, \\
0 & <x<a, 0<y<b, 0<z<c, t>0
\end{aligned}
$$

subject to boundary conditions

$$
\begin{array}{ll}
u_{x}(0, y, z, t)=f_{1}(y, z, t), & u_{x}(a, y, z, t)=f_{2}(y, z, t), \\
u_{y}(x, 0, z, t)=g_{1}(x, z, t), & u_{x}(x, b, z, t)=g_{2}(x, z, t), \\
u_{z}(x, y, 0, t)=h_{1}(x, y, t), & u_{x}(x, y, c, t)=f_{1}(x, y, t),
\end{array}
$$

and the initial conditions

$$
u(x, y, z, 0)=\eta(x, y, z), \quad u_{t}(x, y, z, 0)=\psi(x, y, z)
$$

where $\alpha$ is a parameter describing the fractional derivative, $u_{t}$ is the rate of change of temperature at a point over time. The general response expression contains a parameter describing the order of the fractional derivative that can be varied to obtain various responses. In the case of $0<\alpha \leq 1$, Eq. (1.1) reduces to the fractional heat-like equation with variable coefficients, and it does to a wave-like equation with variable coefficients for $1<\alpha \leq 2$.

\section{Fractional calculus}

We have well-known definitions of a fractional derivative of order $\alpha>0$ such as RiemannLiouville, Grunwald-Letnikow, Caputo and generalized functions approach [1, 22]. The most commonly used definitions are the Riemann-Liouville and Caputo. We give some basic definitions and properties of the fractional calculus theory which are used throughout the paper. 
Definition 2.1 A real function $f(x), x>0$ is said to be in the space $C_{\mu}, \mu \in R$ if there exists a real number $(p>\mu)$ such that $f(x)=x^{p} f_{1}(x)$, where $f_{1}(x) \in C[0, \infty)$, and it is said to be in the space $C_{\mu}^{m}$ iff $f^{m} \in C_{\mu}, m \in N$.

Definition 2.2 The Riemann-Liouville fractional integral operator of order $\alpha \geq 0$ of a function $f \in C_{\mu}, \mu \geq-1$, is defined as

$$
\begin{aligned}
& J_{0}^{v} f(x)=\frac{1}{\Gamma(v)} \int_{0}^{x}(x-t)^{\nu-1} f(t) d t, \quad v>0, \\
& J^{0} f(x)=f(x) .
\end{aligned}
$$

It has the following properties:

For $f \in C_{\mu}, \mu \geq-1, \alpha, \beta \geq 0$, and $\gamma>1$ :

1. $J^{\alpha} J^{\beta} f(x)=J^{\alpha+\beta} f(x)$,

2. $J^{\alpha} J^{\beta} f(x)=J^{\beta} J^{\alpha} f(x)$,

3. $J^{\alpha} x^{\gamma}=\frac{\Gamma(\gamma+1)}{\Gamma(\alpha+\gamma+1)} x^{\alpha+\gamma}$.

The Riemann-Liouville fractional derivative is mostly used by mathematicians, but this approach is not suitable for physical problems of the real world since it requires the definition of fractional order initial conditions, which have no physically meaningful explanation yet. Caputo introduced an alternative definition, which has the advantage of defining integer order initial conditions for fractional order differential equations.

Definition 2.3 The fractional derivative of $f(x)$ in the Caputo sense is defined as

$$
D_{*}^{v} f(x)=J_{a}^{m-v} D^{m} f(x)=\frac{1}{\Gamma(m-v)} \int_{0}^{x}(x-t)^{m-v-1} f^{(m)}(t) d t,
$$

for $m-1<v<m, m \in N, x>0, f \in C_{-1}^{m}$.

Lemma 2.1 If $m-1<\alpha<m, m \in N$ and $f \in C_{\mu}^{m}, \mu \geq-1$, then

$$
\begin{aligned}
& D_{*}^{\alpha} J^{\alpha} f(x)=f(x), \\
& J^{\alpha} D_{*}^{v} f(x)=f(x)-\sum_{k=0}^{m-1} f^{k}\left(0^{+}\right) \frac{x^{k}}{k !}, \quad x>0 .
\end{aligned}
$$

The Caputo fractional derivative is considered here because it allows traditional initial and boundary conditions to be included in the formulation of the problem.

Definition 2.4 For $m$ to be the smallest integer that exceeds $\alpha$, the Caputo time-fractional derivative operator of order $\alpha>0$ is defined as

$$
D_{* t}^{\alpha} u(x, t)=\frac{\partial^{\alpha} u(x, t)}{\partial t^{\alpha}}= \begin{cases}\frac{1}{\Gamma(m-\alpha)} \int_{0}^{t}(t-\xi)^{m-\alpha-1} \frac{\partial^{m} u(x, \xi)}{\partial \xi^{m}} d \xi, & \text { for } m-1<\alpha<m \\ \frac{\partial^{m} u(x, t)}{\partial t^{m}}, & \text { for } \alpha=m \in N .\end{cases}
$$




\section{Differential transform method}

The DTM is applied to the solution of electric circuit problems. The DTM is a numerical method based on the Taylor series expansion which constructs an analytical solution in the form of a polynomial. The traditional high-order Taylor series method requires symbolic computation. However, the DTM obtains a polynomial series solution by means of an iterative procedure. The method is well addressed in [19].

Consider a function of two variables $u(x, y)$, and suppose that it can be represented as a product of two single-variable functions, i.e., $u(x, y)=f(x) g(y)$. Based on the properties of the generalized two-dimensional differential transform [6,23], the function $u(x, y)$ can be represented as

$$
\begin{aligned}
u(x, y) & =\sum_{k=0}^{\infty} F_{\alpha}(k)\left(x-x_{0}\right)^{k \alpha} \sum_{h=0}^{\infty} G_{\beta}(h)\left(y-y_{0}\right)^{h \beta} \\
& =\sum_{k=0}^{\infty} \sum_{h=0}^{\infty} U_{\alpha \beta}(k, h)\left(x-x_{0}\right)^{k \alpha}\left(y-y_{0}\right)^{h \beta},
\end{aligned}
$$

where $0<\alpha, \beta \leq 1, U_{\alpha \beta}(k, h)=F_{\alpha}(k) G_{\beta}(h)$ is called the spectrum of $u(x, y)$. The generalized two-dimensional differential transform of the function $u(x, y)$ is given by

$$
U_{\alpha, \beta}(k, h)=\frac{1}{\Gamma(\alpha k+1) \Gamma(\beta h+1)}\left[\left(D_{* x_{0}}^{\alpha}\right)^{k}\left(D_{* y_{0}}^{\beta}\right)^{h} u(x, y)\right]_{\left(x_{0}, y_{0}\right)},
$$

where $\left(D_{x_{0}}^{\alpha}\right)^{k}=D_{x_{0}}^{\alpha} D_{x_{0}}^{\alpha} \cdots D_{x_{0}}^{\alpha}, k$-times. In the case of $\alpha=1$ and $\beta=1$, the generalized two-dimensional differential transform (3.1) reduces to the classical two-dimensional differential transform [24].

The operators in two-dimensional differential transformation method [24].

Let $U_{\alpha, \beta}(k, h), V_{\alpha, \beta}(k, h)$ and $W_{\alpha, \beta}(k, h)$ be the differential transformations of the functions $u(x, y), v(x, y)$ and $w(x, y)$ :

(a) If $u(x, y)=v(x, y) \pm w(x, y)$, then $U_{\alpha, \beta}(k, h)=V_{\alpha, \beta}(k, h) \pm W_{\alpha, \beta}(k, h)$.

(b) If $u(x, y)=a v(x, y), a \in R$, then $U_{\alpha, \beta}(k, h)=a V_{\alpha, \beta}(k, h)$.

(c) If $u(x, y)=v(x, y) w(x, y)$, then $U_{\alpha, \beta}(k, h)=\sum_{r=0}^{k} \sum_{s=0}^{h} V_{\alpha, \beta}(r, h-s) W_{\alpha, \beta}(k-r, s)$.

(d) If $u(x, y)=\left(x-x_{0}\right)^{n \alpha}\left(y-y_{0}\right)^{m \beta}$, then $U_{\alpha, \beta}(k, h)=\delta(k-n) \delta(h-m)$.

(e) If $u(x, y)=v(x, y) w(x, y) q(x, y)$, then

$$
U_{\alpha, \beta}(k, h)=\sum_{r=0}^{k} \sum_{t=0}^{k-r} \sum_{t=0}^{h} V_{\alpha, \beta}(r, h-s-p) W_{\alpha, \beta}(t, s) Q_{\alpha, \beta}(k-r-t, p) .
$$

(f) If $u(x, y)=D_{x_{0}}^{\alpha} v(x, y), 0<\alpha \leq 1$, then $U_{\alpha, \beta}(k, h)=\frac{\Gamma(\alpha(k+1)+1)}{\Gamma(\alpha k+1)} V_{\alpha, \beta}(k+1, h)$.

(g) If $u(x, y)=f(x) g(y)$ and the function $f(x)=x^{\lambda} h(x)$, where $\lambda>-1, h(x)$ has the generalized Taylor series expansion $h(x)=\sum_{n=0}^{\infty} a_{n}\left(x-x_{0}\right)^{\alpha k}$, and [24],

(i) $\beta<\lambda+1$ and $\alpha$ arbitrary or

(ii) $\beta \geq \lambda+1, \alpha$ arbitrary and $a_{n}=0$ for $n=0,1, \ldots, m-1$, where $m-1<\beta \leq m$.

Then the generalized differential transform (3.2) becomes

$$
U_{\alpha, \beta}(k, h)=\frac{1}{\Gamma(\alpha k+1) \Gamma(\beta h+1)}\left[D_{* x_{0}}^{\alpha k}\left(D_{* y_{0}}^{\beta}\right)^{h} u(x, y)\right]_{\left(x_{0}, y_{0}\right)} .
$$


(h) If $u(x, y)=D_{x_{0}}^{\gamma} v(x, y), m-1<\gamma \leq m$ and $v(x, y)=f(x) g(y)$, then

$$
U_{\alpha, \beta}(k, h)=\frac{\Gamma(\alpha k+\gamma+1)}{\Gamma(\alpha k+1)} V_{\alpha, \beta}(k+\gamma / \alpha, h)
$$

(i) If $u(x, y, t)=D_{* x_{0}}^{\alpha} v(x, y, t), 0<\alpha \leq 1$, then

$$
U_{\alpha, \beta, \gamma}(k, h, m)=\frac{\Gamma(\alpha(k+1)+1)}{\Gamma(\alpha k+1)} V_{\alpha, \beta, m}(k+1, h, m) .
$$

(j) If $u(x, y)=a(x, y) \frac{\partial^{2} v(x, y)}{\partial v^{2}(x, y)}$, then

$$
U(k, h)=\sum_{i=0}^{k} \sum_{j=0}^{h}(k-i+2)(k-i+1) A(i, j) U(k-i+2, h-j) .
$$

The proofs of some properties can be found in [24].

\section{Examples}

Example 4.1 We consider the following one-dimensional fractional heat-like problem:

$$
D_{t}^{\alpha} u=\frac{1}{2} x^{2} u_{x x}, \quad 0<x<1,0<\alpha \leq 1, t>0,
$$

subject to the boundary conditions

$$
u(0, t)=0, \quad u(1, t)=e^{t}
$$

with the initial condition

$$
u(x, 0)=x^{2} .
$$

The exact solution $(\alpha=1)$ was found to be [4]

$$
u(x, t)=x^{2} e^{t} .
$$

Taking the differential transform of Eq. (4.1), by using the property, we have

$$
\begin{aligned}
& \frac{\Gamma(\alpha(h+1)+1)}{\Gamma(\alpha h+1)} U_{\alpha}(k, h+1) \\
& =\frac{1}{2} \sum_{i=0}^{k} \sum_{j=0}^{h}(k-i+2)(k-i+1) A(i, j) U_{\alpha}(k-i+2, h-j) .
\end{aligned}
$$

We start with the initial condition that was given by Eq. (4.2). By using the above formula (4.4), we can obtain the other components by using mathematical tools MAPLE package as follows:

$$
u(x, t)=x^{2}\left[1+\frac{t^{\alpha}}{\Gamma(1+\alpha)}+\frac{t^{2 \alpha}}{\Gamma(1+2 \alpha)}+\frac{t^{3 \alpha}}{\Gamma(1+3 \alpha)}+\frac{t^{4 \alpha}}{\Gamma(1+4 \alpha)}+\cdots\right]
$$


So, the solution for the standard heat-like equation $(\alpha=1)$ is given by [4]

$$
u(x, t)=x^{2}\left[1+t+\frac{t^{2}}{2}+\frac{t^{3}}{3 !}+\frac{t^{4}}{4 !}+\cdots\right]
$$

Example 4.2 In this example, we consider the two-dimensional fractional heat-like equation

$$
D_{t}^{\alpha} u=u_{x x}+u_{y y}, \quad 0<x, y<2 \pi, 0<\alpha \leq 1, t>0,
$$

subject to the boundary conditions

$$
\begin{array}{ll}
u(0, y, t)=0, & u(2 \pi, y, t)=0, \\
u(x, 0, t)=0, & u(x, 2 \pi, t)=0,
\end{array}
$$

and the initial condition

$$
u(x, y, 0)=\sin x \sin y .
$$

The exact solution $(\alpha=1)$ was found to be [4]

$$
u(x, y, t)=e^{-2 t} \sin x \sin y .
$$

Taking the differential transform of Eq. (4.5), by using the related property, we have

$$
\begin{aligned}
& \frac{\Gamma(\alpha(m+1)+1)}{\Gamma(\alpha m+1)} U_{\alpha}(k, h, m+1) \\
& \quad=(k+2)(k+1) U_{\alpha}(k+2, h, m)+(h+2)(h+1) U_{\alpha}(k, h+2, m) .
\end{aligned}
$$

We start with the initial condition that was given by Eq. (4.6). The solution for the fractional heat-like Eq. (4.5) in a series form is given by

$$
\begin{aligned}
u(x, y, t)= & \sin x \sin y\left[1-2 \frac{t^{\alpha}}{\Gamma(1+\alpha)}+4 \frac{t^{2 \alpha}}{\Gamma(1+2 \alpha)}\right. \\
& \left.-8 \frac{t^{3 \alpha}}{\Gamma(1+3 \alpha)}+16 \frac{t^{4 \alpha}}{\Gamma(1+4 \alpha)}+\cdots\right] .
\end{aligned}
$$

For the special case $(\alpha=1)$, we can reproduce the series solution of [4], and the solution in a closed form

$$
u(x, y, t)=e^{-2 t} \sin x \sin y .
$$

Example 4.3 Consider the following three-dimensional fractional heat-like equation:

$$
D_{t}^{\alpha} u=x^{4} y^{4} z^{4}+\frac{1}{36}\left[x^{2} u_{x x}+y^{2} u_{y y}+z^{2} u_{z z}\right], \quad 0<x, y, z<1,0<\alpha \leq 1, t>0,
$$


subject to the boundary conditions

$$
\begin{array}{ll}
u(0, y, z, t)=0, & u(1, y, z, t)=y^{4} z^{4}\left(e^{t}-1\right), \\
u(x, 0, z, t)=0, & u(x, 1, z, t)=x^{4} z^{4}\left(e^{t}-1\right), \\
u(x, y, 0, t)=0, & u(x, y, 1, t)=y^{4} x^{4}\left(e^{t}-1\right),
\end{array}
$$

and the initial condition

$$
u(x, y, z, 0)=0
$$

The exact solution $(\alpha=1)$ was found to be [4]

$$
u(x, y, z, t)=y^{4} x^{4} z^{4}\left(e^{t}-1\right) .
$$

Taking the differential transform of Eq. (4.7), by using the related property, we have

$$
\begin{aligned}
& \frac{\Gamma(\alpha(l+1)+1)}{\Gamma(\alpha l+1)} U_{\alpha}(k, h, m, l+1) \\
& =\delta(k-4) \delta(h-4) \delta(m-4) \\
& \quad+\frac{1}{36}\left[U_{\alpha} k(k-1)(k, h, m, l)+U_{\alpha} h(h-1)(k, h, m, l)+U_{\alpha} m(m-1)(k, h, m, l)\right] .
\end{aligned}
$$

We start with the initial condition. The solution for the fractional heat-like Eq. (4.7) in a series form is given by

$$
u(x, y, z, t)=x^{4} y^{4} z^{4}\left[\frac{t^{\alpha}}{\Gamma(1+\alpha)}+\frac{t^{2 \alpha}}{\Gamma(1+2 \alpha)}+\frac{t^{3 \alpha}}{\Gamma(1+3 \alpha)}+\frac{t^{4 \alpha}}{\Gamma(1+4 \alpha)}+\cdots\right]
$$

For the special case $(\alpha=1)$, we can reproduce the series solution of [4], and the solution in a closed form

$$
u(x, y, z, t)=y^{4} x^{4} z^{4}\left(e^{t}-1\right) .
$$

We also conclude that our approximate solutions are in good agreement with the exact values. Both DTM and ADM have highly accurate solutions, but DTM has an easier way than ADM. We can solve the equation directly without calculating the Adomian polynomials.

Example 4.4 Next, we consider the one-dimensional fractional wave-like equation

$$
D_{t}^{\alpha} u=\frac{1}{2} x^{2} u_{x x}, \quad 0<x<1,1<\alpha \leq 2, t>0,
$$

subject to the boundary conditions

$$
u(0, t)=0, \quad u(1, t)=1+\sinh t,
$$


and the initial conditions

$$
u(x, 0)=0, \quad u_{t}(x, 0)=x^{2} .
$$

Taking the differential transform of Eq. (4.8), by using the related property, we have

$$
\frac{\Gamma(\alpha h+\gamma+1)}{\Gamma(\alpha h+1)} U_{\alpha}\left(k, h+n_{1}\right)=\frac{1}{2} k(k-1) U_{\alpha}(k, h) .
$$

In the case of $\alpha=2$ in Eq. (4.8), we start with the initial conditions that were given by Eq. (4.9).

The solution for the fractional wave-like Eq. (4.8) in a series form is given by

$$
u(x, t)=x+x^{2}\left[t+\frac{t^{3}}{3 !}+\frac{t^{5}}{5 !}+\frac{t^{7}}{7 !}+\frac{t^{9}}{9 !}+\cdots\right]
$$

and the exact [3] solution for this special case is

$$
u(x, t)=x+x^{2} \sinh t .
$$

Example 4.5 We consider the three-dimensional fractional wave-like equation

$$
D_{t}^{\alpha} u=x^{2}+y^{2}+z^{2}+\frac{1}{2}\left[x^{2} u_{x x}+y^{2} u_{y y}+z^{2} u_{z z}\right], \quad 0<x, y, z<1,1<\alpha \leq 2, t>0,
$$

subject to the boundary conditions

$$
\begin{aligned}
& u(0, y, z, t)=y^{2}\left(e^{t}-1\right)+z^{2}\left(e^{-t}-1\right), \\
& u(1, y, z, t)=\left(1+y^{2}\right)\left(e^{t}-1\right)+z^{2}\left(e^{-t}-1\right), \\
& u(x, 0, z, t)=x^{2}\left(e^{t}-1\right)+z^{2}\left(e^{-t}-1\right), \\
& u(x, 1, z, t)=\left(1+x^{2}\right)\left(e^{t}-1\right)+z^{2}\left(e^{-t}-1\right), \\
& u(x, y, 0, t)=\left(x^{2}+y^{2}\right)\left(e^{t}-1\right), \quad u(x, y, 1, t)=\left(x^{2}+y^{2}\right)\left(e^{t}-1\right)+\left(e^{-t}-1\right),
\end{aligned}
$$

and the initial conditions

$$
u(x, y, z, 0)=0, \quad u_{t}(x, y, z, 0)=x^{2}+y^{2}-z^{2} .
$$

The exact solution $(\alpha=2)$ was found to be [3]

$$
u(x, y, z, t)=\left(x^{2}+y^{2}\right) e^{t}+z^{2} e^{-t}-\left(x^{2}+y^{2}+z^{2}\right) .
$$

Taking the differential transform of Eq. (4.10), by using the related property, we have

$$
\begin{aligned}
& \frac{\Gamma(\alpha l+\gamma+1)}{\Gamma(\alpha l+1)} U_{\alpha}\left(k, h, m, l+n_{1}\right) \\
& =\delta(k-2)+\delta(h-2)+\delta(m-2) \\
& \quad+\frac{1}{2}\left[U_{\alpha} k(k-1)(k, h, m, l)+U_{\alpha} h(h-1)(k, h, m, l)+U_{\alpha} m(m-1)(k, h, m, l)\right] .
\end{aligned}
$$


In case of $\alpha=2$ in Eq. (4.10), we start with the initial conditions that were given by Eq. (4.11). The solution for the fractional wave-like Eq. (4.10) in a series form is given by

$$
u(x, y, z, t)=\left(x^{2}+y^{2}\right)\left[t+\frac{t^{2}}{2 !}+\frac{t^{3}}{3 !}+\frac{t^{4}}{4 !}+\frac{t^{5}}{5 !}+\cdots\right]+z^{2}\left[-t+\frac{t^{2}}{2 !}-\frac{t^{3}}{3 !}+\frac{t^{4}}{4 !}-\frac{t^{5}}{5 !}+\cdots\right]
$$

We can reproduce the series solution of [3], and the solution in a closed form

$$
u(x, y, z, t)=\left(x^{2}+y^{2}\right) e^{t}+z^{2} e^{-t}-\left(x^{2}+y^{2}+z^{2}\right) .
$$

\section{Conclusions}

The application of the differential transform method (DTM) has been successfully employed to obtain the approximate solution of the fractional heat-like and wave-like equations with variable coefficients. The method was used in a direct way without using linearization, perturbation or restrictive assumptions. The procedure presented to solve the fractional heat-like and wave-like equations is the same as that for standard heat-like and wave-like equations, and in special cases of $\alpha=1$ and $\alpha=2$, the general solution reduces to the heat-like and wave-like solutions.

\section{Competing interests}

The author declares that they have no competing interests.

Received: 28 September 2012 Accepted: 2 November 2012 Published: 14 November 2012

\section{References}

1. Podlubny, I: Fractional Differential Equations. Academic Press, San Diego (1999)

2. Schneider, WR, Wyss, W: Fractional diffusion and wave equations. J. Math. Phys. 30, 134-144 (1989)

3. Wazwaz, AM, Gorguis, A: Exact solutions of heat-like and wave-like equations with variable coefficients. Appl. Math. Comput. 149(1), 15-29 (2004)

4. Momani, S: Analytical approximate solution for fractional heat-like and wave-like equations with variable coefficients using the decomposition method. Appl. Math. Comput. 165(2), 459-472 (2005)

5. Odibat, ZM, Momani, S: Application of variational iteration method to nonlinear differential equations of fractional order. Int. J. Nonlinear Sci. Numer. Simul. 7(1), 27-34 (2006)

6. Bildik, $N$, Konuralp, A: The use of variational iteration method, differential transform method and Adomian decomposition method for solving different types of nonlinear partial differential equations. Int. J. Nonlinear Sci. Numer. Simul. 7, 65-70 (2006)

7. Sweilam, NH, Khader, MM: Variational iteration method for one dimensional nonlinear thermo-elasticity. Chaos Solitons Fractals 32, 145-149 (2007)

8. Momani, S, Odibat, Z: Numerical comparison of methods for solving linear differential equations of fractional order. Chaos Solitons Fractals 31(5), 1248-1255 (2007)

9. Soliman, AA: A numerical simulation and explicit solutions of KdV-Burgers' and Lax's seventh-order KdV equations. Chaos Solitons Fractals 29, 294-302 (2006)

10. $\mathrm{Xu}, \mathrm{H}$, Cang, J: Analysis of a time fractional wave-like equation with the homotopy analysis method. Phys. Lett. A 372 1250-1255 (2008)

11. $\mathrm{He}, \mathrm{JH}$ : Approximate analytical solution for seepage flow with fractional derivatives porous media. Comput. Methods Appl. Mech. Eng. 167, 57-68 (1998)

12. Shou, $\mathrm{DH}, \mathrm{He}, \mathrm{J}-\mathrm{H}$ : Beyond Adomian method: the variational iteration method for solving heat-like equations with variable coefficients. Phys. Lett. A 372, 233-237 (2008)

13. Molliq, RY, Noorani, MSM, Hashim, I: Variational iteration method for fractional heat- and wave-like equations. Nonlinear Anal., Real World Appl. 10(3), 1854-1869 (2009)

14. Zhou, JK: Differential Transformation and Its Applications for Electrical Circuits. Huazhong University Press, Wuhan (1986)

15. Arikoglu, A, Ozkol, I: Solution of fractional differential equations by using differential transform method. Chaos Solitons Fractals 34, 1473-1481 (2007)

16. Hilfer, R (ed.): Applications of Fractional Calculus in Physics. Academic Press, Orlando (1999)

17. Odibat, Z, Momani, S: Numerical methods for nonlinear partial differential equations of fractional order. Appl. Math. Model. 32, 28-39 (2008)

18. Kurulay, M, Bayram, M: Approximate analytical solution for the fractional modified KdV by differential transform method. Commun. Nonlinear Sci. Numer. Simul. 15(7), 1777-1782 (2010) 
19. Odibat, Z, Momani, S, Erturk, VS: Generalized differential transform method: for solving a space- and time-fractional diffusion-wave equation. Phys. Lett. A 370, 379-387 (2007)

20. Hristov, J: Heat-balance integral to fractional (half-time) heat diffusion sub-model. Therm. Sci. 14(2), 291-316 (2010)

21. Histov, J: Starting radial subdiffusion from a central point through a diverging medium (a sphere): heat-balance integral method. Therm. Sci. 15(1), S5-S20 (2011)

22. Caputo, M: Linear models of dissipation whose $Q$ is almost frequency independent. Part II. Geophys. J. R. Astron. Soc. $13,529-539$ (1967)

23. Abdel-Halim Hassan, IH: Comparison differential transformation technique with Adomian decomposition method for linear and nonlinear initial value problems. Chaos Solitons Fractals 36, 53-65 (2008)

24. Momani, S, Odibat, Z: A novel method for nonlinear fractional partial differential equations: combination of DTM and generalized Taylor's formula. J. Comput. Appl. Math. 220, 85-95 (2008)

doi:10.1186/1687-1847-2012-198

Cite this article as: Secer: Approximate analytic solution of fractional heat-like and wave-like equations with variable coefficients using the differential transforms method. Advances in Difference Equations 2012 2012:198.

\section{Submit your manuscript to a SpringerOpen ${ }^{\odot}$ journal and benefit from:}

- Convenient online submission

- Rigorous peer review

- Immediate publication on acceptance

- Open access: articles freely available online

- High visibility within the field

- Retaining the copyright to your article 